\title{
Cortical Actin Alteration at the Matrix-Side Cytoplasm in Lung Adenocarcinoma Cells and Its Significance in Invasion
}

\author{
Shohei Tomii ${ }^{a}$ Takumi Akashi $^{a}$ Noboru Ando ${ }^{a}$ Tomoki Tamura ${ }^{a}$ \\ Akira Sakurai $^{a}$ Asami Terada ${ }^{a}$ Asuka Furukawa ${ }^{a}$ Yoshimi Suzukia \\ Kou Kayamori $^{\mathrm{b}}$ Kei Sakamoto ${ }^{\mathrm{b}}$ Hironori Ishibashic ${ }^{\mathrm{c}}$ Yoshinobu Eishia \\ Departments of a Pathology, ${ }^{\text {b }}$ Oral Pathology and ${ }^{\mathrm{C}}$ Thoracic Surgery, Graduate School of Medical and \\ Dental Sciences, Tokyo Medical and Dental University, Tokyo, Japan
}

\section{Keywords}

Lung · Adenocarcinoma - Cortical actin · Nonmuscle myosin · Invasion · Migration · Cdc42 - Blebbistatin

\begin{abstract}
Objectives: Cortical actin is a thin layer of filamentous (F-) actin that lies beneath the plasma membrane, and its role in pathophysiology remains unclear. We investigated the subcellular localization of cortical actin by the histopathological and experimental studies of lung adenocarcinomas. Materials and Methods: The subcellular localization of cortical actin was studied in surgically resected lung adenocarcinomas tissues and in 3-dimensionally cultured lung adenocarcinoma A549 cells. Results: In normal type II alveolar cells and the bronchiolar epithelium, cortical actin was localized to the apical-side cytoplasm. In invasive adenocarcinoma cells, cortical actin was frequently localized to the matrix side. The degree of cortical actin localized to the matrix side was associated with the loss of basement membrane and a poor prognosis. In A549 cell spheroids cultured in a type I collagen and basement membrane extract Matrigel ${ }^{\mathrm{TM}}$ mixed gel, cortical F-actin was localized to the matrix side with phosphorylated myosin light chain. Super-resolution and electron mi-
\end{abstract}

\section{KARGER}

(C) 2016 S. Karger AG, Basel

E-Mail karger@karger.com

www.karger.com/pat croscopy results suggest that compact wrinkling of the plasma membrane by myosin-mediated F-actin contraction is an explanation for cortical actin accumulation at the matrix side. The myosin II inhibitor blebbistatin suppressed the 3-dimensional collective migration of A549 cells induced by constitutively active Cdc42 and MT1-MMP. Conclusion: Cortical actin accumulation at the matrix-side cytoplasm of cancer cells occurs in invasive lung adenocarcinomas and it possibly participates in the migration of cancer cells through myosin-mediated contraction.

(c) 2016 S. Karger AG, Basel

\section{Introduction}

Actin plays a key role in cell migration through both polymerization by its nucleators and contraction with myosin motor proteins. In a 2-dimensional (2D) environment, migration is dependent on lamellipodia formed by actin polymerization [1]. In a 3-dimensional (3D) environment, distinct modes of migration such as single-cell

\section{S.T. and T.A. contributed equally to this work.}

Takumi Akashi

Department of Pathology, Graduate School of Medical and Dental Sciences Tokyo Medical and Dental University

1-5-45, Yushima, Bunkyo-ku, Tokyo 113-8519 (Japan)

E-Mail akashi.path@tmd.ac.jp 
mesenchymal, amoeboid migration, and multicellular collective cell migration occur. Mesenchymal migration has some parallels with 2D migration [2] and pseudopodia-based actin polymerization with additional matrix proteolysis generates its main propulsion force. In contrast, contraction of cortical actin generates the main propulsion force in amoeboid migration both by bleb-dependent and independent manners $[3,4]$. Contraction of cortical actomyosin is also considered crucial in the collective migration of squamous cell carcinoma cells [5].

Cortical actin is a thin layer of filamentous (F-)actin that lies beneath the plasma membrane with nonmuscle myosin and actin-binding proteins; it participates in cellular differentiation, morphogenesis, cytokinesis, and migration [6]. The pathophysiology of the actin cortex has not been thoroughly studied [7]. Cortical F-actin is distributed throughout the cellular cortex; however, it shows a specific subcellular localization to the apical domain apart from the basal/matrix side in polarized epithelial cells of a developing mouse neural tube [8] and a Drosophila egg chamber [9]. In the amnioserosal epithelium of Drosophila, the distinct actin cortex is present at the apical surface and its contraction participates in apical constriction [10]. In contrast, in the squamous cell carcinoma A431 cells, the actomyosin filament is localized to the cell cortex in contact with extracellular matrix and it participates in collective cell migration [5]. In our previous study, subcellular localization of the actin-binding proteins actinin-1 and cortactin showed translocation from the apical side to the matrix side facing surrounding interstitium in lung cancer cells and it significantly correlated to cancer cell invasion [11]. Accordingly, we hypothesized that the subcellular translocation of F-actin possibly occurs in cancer cells and this participates in invasion.

In this study, we initially used immunohistochemistry to study the subcellular localization of $\beta$-actin in clinical samples of lung adenocarcinomas and verify its correlation to invasion, metastasis, and prognosis. Next, we experimentally studied the participation of cortical actin in cancer cell migration using an in vitro 3D model of cancer cell migration. We also discuss how the matrix-side cortical actin can participate in the invasion of cancer cells.

\section{Materials and Methods}

Patients

Between 1999 and 2007, 109 cases of adenocarcinomas that were surgically resected in the Tokyo Medical and Dental University Hospital were examined (online suppl. Table 1; for all online suppl. material, see www.karger.com/doi/10.1159/000452838). The cases fulfilled the following prerequisites: each patient underwent a lobectomy or segmentectomy with lymph node dissection, no patient underwent preoperative chemotherapy, and follow-up data from each patient was available. Each lesion was fixed in formalin and embedded in paraffin. This study was conducted in accordance with the amended Declaration of Helsinki and approved by the Internal Review Board of Bioethics of Tokyo Medical and Dental University (registry No. 727 and 2028). Although written informed consent was not obtained from the patients, the ethics committee approved waiver of specific informed consent in accordance with amended Ethical Guidelines for Clinical Studies provided by the Ministry of Health, Labor and Welfare of Japan (July $31,2008)$. This research plan was disclosed in a poster format in the hospital to ensure that patients had the opportunity to refuse research use and this disclosure was substituted for informed consent, and the ethics committee approved this consent procedure.

\section{Histological Evaluation}

Adenocarcinomas were classified into adenocarcinoma in situ (AIS), minimally invasive adenocarcinoma (MIA), and lepidic, acinar, papillary, micropapillary, solid, and invasive mucinous subtypes according to the WHO classification criteria [12]. Tumor stages were determined according to the staging manual of the International Association of the Study of Lung Cancer [13].

\section{Immunohistochemical Examination}

The antibodies used were as follows: $\beta$-actin $(1: 4,000$, EP1123Y, Epitomics, Burlingame, CA, USA), laminin-511/C1 (1:100, sc59846, Santa Cruz Biotechnology, Santa Cruz, CA, USA,), and phospho-MLC2 (myosin light chain 2; $\times 200$, 3674, Cell Signaling Technology, Danvers, MA, USA). Deparaffinized sections were heated in an antigen retrieval buffer (Nichirei Bioscience, Tokyo, Japan) at $98^{\circ} \mathrm{C}$ for $40 \mathrm{~min}$ or reacted with a protease for $30 \mathrm{~min}$ (laminin-C1). The slides were then treated with methanol containing $0.3 \%(\mathrm{v} / \mathrm{v})$ hydrogen peroxide for $10 \mathrm{~min}$ and reacted with primary antibodies, ABC detection kit (Vector, Burlingame, CA, USA), and diaminobenzidine in succession. For immunofluorescence, anti-mouse IgG-Alexa 488 and anti-rabbit IgG-Alexa 546 were used.

From each case, we selected 1 paraffin block which was composed of noninvasive/lepidic and invasive subtypes with the appropriate proportion in order to make it representative of each case. The scoring of immunohistochemical findings was done on paraffin sections. As a proportion of positive or negative cells showed a more prominent difference among the examined cases and this was easier to quantify than the strength of staining, grading was according to the proportion of positive cells ( $\beta$-actin) or negative cells (laminin-C1) [11]. The following scale was used: none as " 0 ", up to $5 \%$ as " 1 ", $5 \%$ to one-third as " 2 ", one- to twothirds as " 3 ", two-thirds to $95 \%$ as " 4 ", $95 \%$ to less than $100 \%$ as " 5 ", and $100 \%$ as " 6 ". Grades of " 0 " and " 1 " were classified as low and " $2-6$ " as high.

For the staining of actin on frozen sections, formalin-fixed tissues were immersed with PBS containing 20\% sucrose, embedded in OCT compound (Tissue Tek, Torrance, CA, USA), and frozen in liquid nitrogen. Frozen sections were reacted with phalloidinAlexa 488 or phalloidin-Alexa 555 (1:100 Cytoskeleton, Denver, CO, USA). Frozen sections were reacted with methanol for $10 \mathrm{~min}$ before the immunostaining of $\beta$-actin. Fluorescence images were
172
Pathobiology 2017;84:171-183

DOI: $10.1159 / 000452838$
Tomii et al. 
acquired on an inverted confocal microscope (Fv1200, Olympus, Tokyo, Japan) and analyzed with software OSR (Olympus) for the acquisition of super-resolution images.

\section{Statistical Analysis}

Correlations between the patients' clinicopathological factors and immunohistochemical results were assessed by the MannWhitney $U$ test. Tumor-specific survival curves and disease-free survival curves were analyzed by the Kaplan-Meier method, and the statistical significance of differences was determined by logrank test. In the multivariate Cox proportional hazards regression analysis, any clinicopathological factors with a $p$ value $<0.05$ by univariate analysis were included. One patient who died of other diseases was excluded from the analysis of survival. The statistical analysis was performed using StatFlex v.6.0 (Artech, Osaka, Japan).

\section{Preparation of Lentiviral Particles}

Constitutively active myc-Cdc42-Q61L (12974, Addgene, Cambridge, MA, USA), dominant negative myc-Cdc42-T17L (12973, Addgene), DDK-tagged MT1-MMP (Origene, Rockville, MD, USA), and Lifeact-GFP [14] were cloned into either CS-IIEF-RfA (Cdc42, provided by Hiroyuki Miyoshi, RIKEN, Tsukuba, Japan) or CSII-MCS-CMV vector (MT1-MMP and Lifeact-GFP, RIKEN). Lentiviral particles were produced with HEK293T cells and Lenti-X packaging kit (Clontech, Mountain View, CA, USA) according to the manufacturer's protocol. Viral particle-containing supernatants were passed through a $0.45-\mu \mathrm{m}$ filter, concentrated 10 -fold with Lenti-X concentrator (Clontech), aliquoted, and stored at $-80^{\circ} \mathrm{C}$.

Cell Culture in 3D Gels and Lentiviral Infection

A549 lung adenocarcinoma cells was purchased from the Japanese Cancer Research Source Bank. Cells were cultured in Dulbecco's modified Eagle's medium (DMEM) supplemented with $10 \%$ fetal calf serum (FCS). Between 2,000 and 5,000 cells were suspended in $50 \mu \mathrm{L}$ of type I collagen gel (final concentration of 2.0 $\mathrm{mg} / \mathrm{mL}$, Nitta Gelatin, Tokyo, Japan) containing growth factorreduced Matrigel ${ }^{\mathrm{TM}}$ (final concentration of $150 \mu \mathrm{g} / \mathrm{mL}$, Corning, Tewksbury, MA, USA) [5] and supplemented with DMEM. Next, the cells were plated in a well of a 96-well optical plate (Thermo Fisher Scientific, Rochester, NY, USA) and overlaid with $100 \mu \mathrm{L}$ of DMEM/FCS after a $30-\mathrm{min}$ incubation at $37^{\circ} \mathrm{C}$. The cells were fed every 2 days for 8 days. Cells in type I collagen and Matrigel mixed gel were infected with $25 \mu \mathrm{L}$ of lentivirus-containing medium for $15 \mathrm{~h}$ on the fifth day. Blebbistatin (Enzo Life Science, Farmingdale, NY, USA) was used at $50 \mu \mathrm{M}$ and added to the cells on the sixth day, $15 \mathrm{~h}$ after lentiviral infection. For culture in Matrigel, $30 \mu \mathrm{L}$ of Matrigel was plated and a suspension of $2,000-5,000$ cells $/ 100 \mu \mathrm{L}$ of DMEM/FCS with lentiviral particles was overlaid. The DMEM/ FCS was removed on the next day, $20 \mu \mathrm{L}$ of Matrigel was overlaid, and $100 \mu \mathrm{L}$ of DMEM/FCS was then overlaid after a 30-min incubation at $37^{\circ} \mathrm{C}$.

Image Analysis of A549 Cells in 3D Culture

Gels were fixed with paraformaldehyde, washed with PBS containing $0.25 \%$ Tween-20, and then stained with phalloidin and 4',6-diamidino-2-phenylindole (DAPI; Wako, Osaka, Japan). Fluorescence images were acquired using $10 \times \mathrm{O} / 0.40$ or $60 \times \mathrm{O} / 1.40$ objectives on an inverted confocal microscope

Cortical Actin in Lung Adenocarcinoma
(Fv1200) and analyzed using FV10-ASW software (Olympus). For the quantification of phalloidin fluorescence, line tools measured the fluorescence intensities at more than 10 points from each object, each intensity on a line was averaged, and the cortex/ cytoplasm ratio was calculated and regarded as the measured value [6]. For immunocytochemistry, 3D-cultured A549 cells were embedded in paraffin and processed similarly to immunohistochemistry. In order to confirm the specificity of the anti-phosphorylated MLC2 antibody, the section was pretreated with lambda phosphatase (400 units/section, BioAcademia, Osaka, Japan) at $30^{\circ} \mathrm{C}$ for $60 \mathrm{~min}$. Phosphorylated MLC was regarded as positive when more than a quarter of the matrix-side circumference of a spheroid was stained. For electron microscopy, A549 cells were fixed with $2.5 \%$ glutaraldehyde, routinely processed, and observed by a transmission electron microscope (H-7200, Hitachi, Tokyo, Japan).

Quantification of Migration of A549 Cells in 3D Gels

The migration of A549 cells in 3D gels was measured by the nuclear position of A549 cells in order to exclude the effect of elongation of cytoplasmic spikes. The maximum axial lengths of more than 50 colonies in each experimental condition were measured on an inverted confocal microscope and the degree of migration was represented by their average.

\section{Cell Growth Assay}

To assay cell growth, 2,000 A549 cells were seeded on a well of a 96-well plate, incubated with 80-90 $\mu \mathrm{L}$ of DMEM/FCS and 10-20 $\mu \mathrm{L}$ of lentivirus-containing medium for $15 \mathrm{~h}$, and then incubated with DMEM/FCS containing blebbistatin for $48 \mathrm{~h}$. Next, the medium was replaced with $100 \mu \mathrm{L}$ of DMEM/FCS containing $10 \mu \mathrm{L}$ of Cell Counting Kit-8 (Dojindo, Osaka, Japan), incubated for 30 $\mathrm{min}$, and the optical density at $450 \mathrm{~nm}$ was measured.

\section{RNA Extraction and RT-PCR}

The preparation of mRNA from A549 and normal airway epithelial cells, as well as the quantification of MT1-MMP mRNA, was similar to a previous description [11].

\section{Results}

\section{Cortical Actin Distribution in Normal and Lung Adenocarcinoma Cells}

In normal type II alveolar cells and the bronchiolar epithelium, $\beta$-actin showed localization to the apical- and intercellular-side cytoplasm, but seldom showed matrixside localization (Fig. 1a, b). In AIS, localization of $\beta$-actin was found in apical-side cytoplasm and rarely found in the matrix-side cytoplasm (Fig. 1c, d). The basement membrane represented by laminin-C1 was well preserved (Fig. 1e). In invasive acinar adenocarcinoma cells, $\beta$-actin was frequently observed not only in the apical-side cytoplasm, but also in the matrix-side cytoplasm beneath the basal plasma membrane facing surrounding interstitium (Fig. 1f, g). Most of the matrix-side actin showed a linear

Pathobiology 2017;84:171-183 
Fig. 1. Subcellular localization of $\beta$-actin and basement membrane laminin in normal alveolar, bronchiolar cells, AIS, and invasive acinar adenocarcinoma. Subcellular localization of $\beta$-actin (a-d, $\mathbf{f}, \mathbf{g})$, and laminin-C1 $(\mathbf{e}, \mathbf{h})$ in normal alveolar (a) and bronchiolar cells (b), AIS (c-e), and invasive acinar adenocarcinoma $(\mathbf{f}-\mathbf{h})$. Original magnification, $\times 480(\mathbf{a}, \mathbf{b}), \times 180(\mathbf{c}, \mathbf{e}, \mathbf{f}, \mathbf{h})$, $\times 540(\mathbf{d}, \mathbf{g})$. Arrowheads show the localization of $\beta$-actin to the matrix-side cytoplasm of cancer cells. The arrow $(\mathbf{g})$ shows localization of $\beta$-actin also at the apical-side cytoplasm.

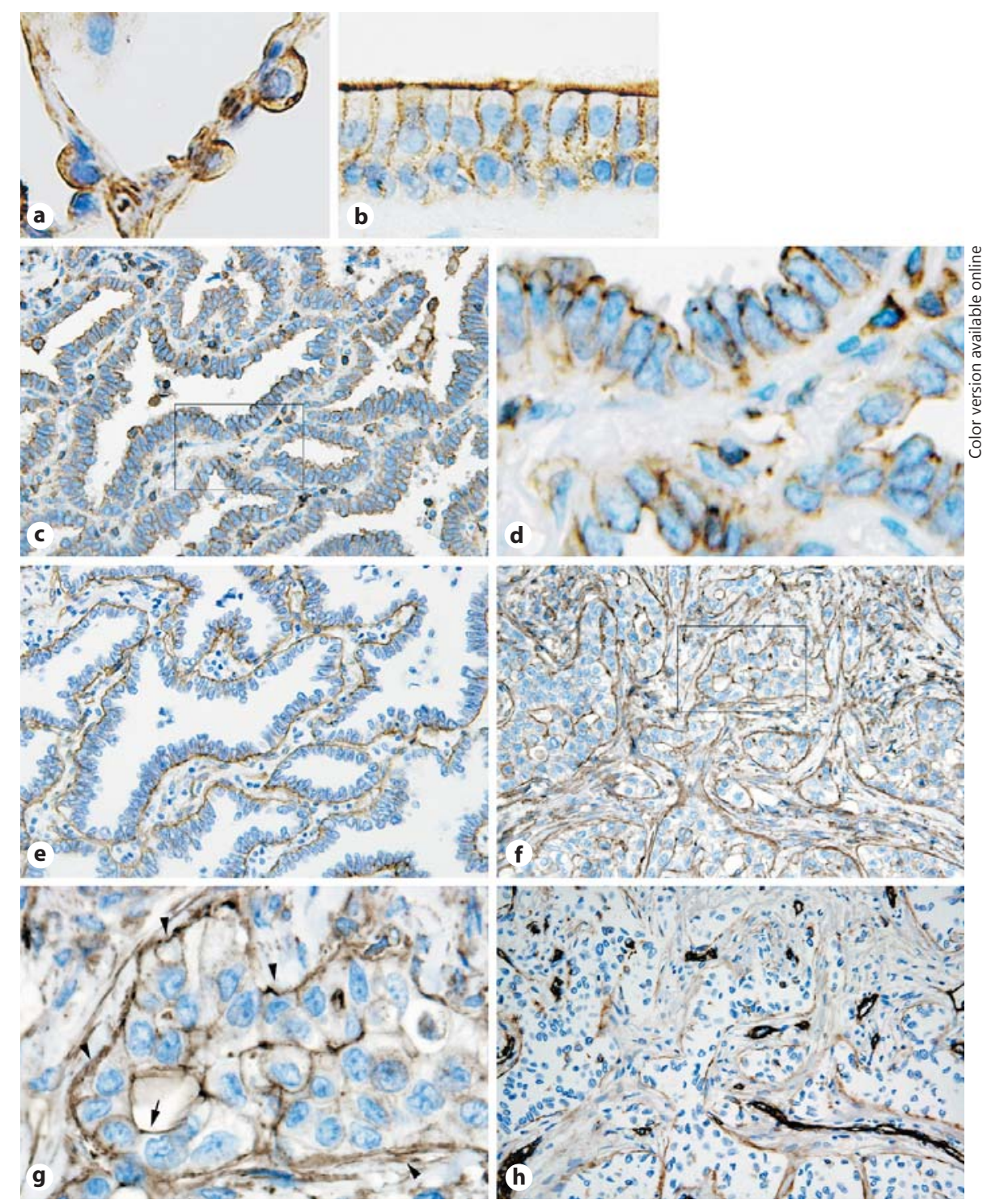

pattern extending over the plural cells. The disruption or absence of laminin-C1 was frequently observed and this correlated with the matrix-side localization of $\beta$-actin (Fig. 1h; online suppl. Fig. 1A). Matrix-side localization of $\beta$-actin was also observed in invasive papillary, micropapillary, and solid adenocarcinomas (Fig. 2a-c). Both in MIAs and lepidic adenocarcinomas, matrix-side localization of $\beta$-actin was observed mainly in the invasive components, and also found in the lepidic components to a lesser degree (Fig. 2d-h). Because of the limited amount of invasive components as well as lesser degree of actin alteration in the lepidic components, the degree of matrix-side localization of $\beta$-actin in MIA was intermediate between those of AIS and invasive adenocarcinomas (online suppl. Fig. 1B-D).

The anti- $\beta$-actin antibody used in this study can react with both monomeric and F-actin. Accordingly, we compared the immunohistochemical distribution of $\beta$-actin and F-actin visualized by phalloidin on frozen sections. In normal bronchiolar cells, the staining of both $\beta$-actin and phalloidin was localized in apical-side cytoplasm (online suppl. Fig. 2A, B). In invasive acinar adenocarcinoma cells, the staining of both $\beta$-actin and phalloidin was found in the matrix-side cytoplasm of cancer cells (online suppl. Fig. 2C, D). The distribution of $\beta$-actin and F-actin-binding Lifeact by fluorescent double immuno- 


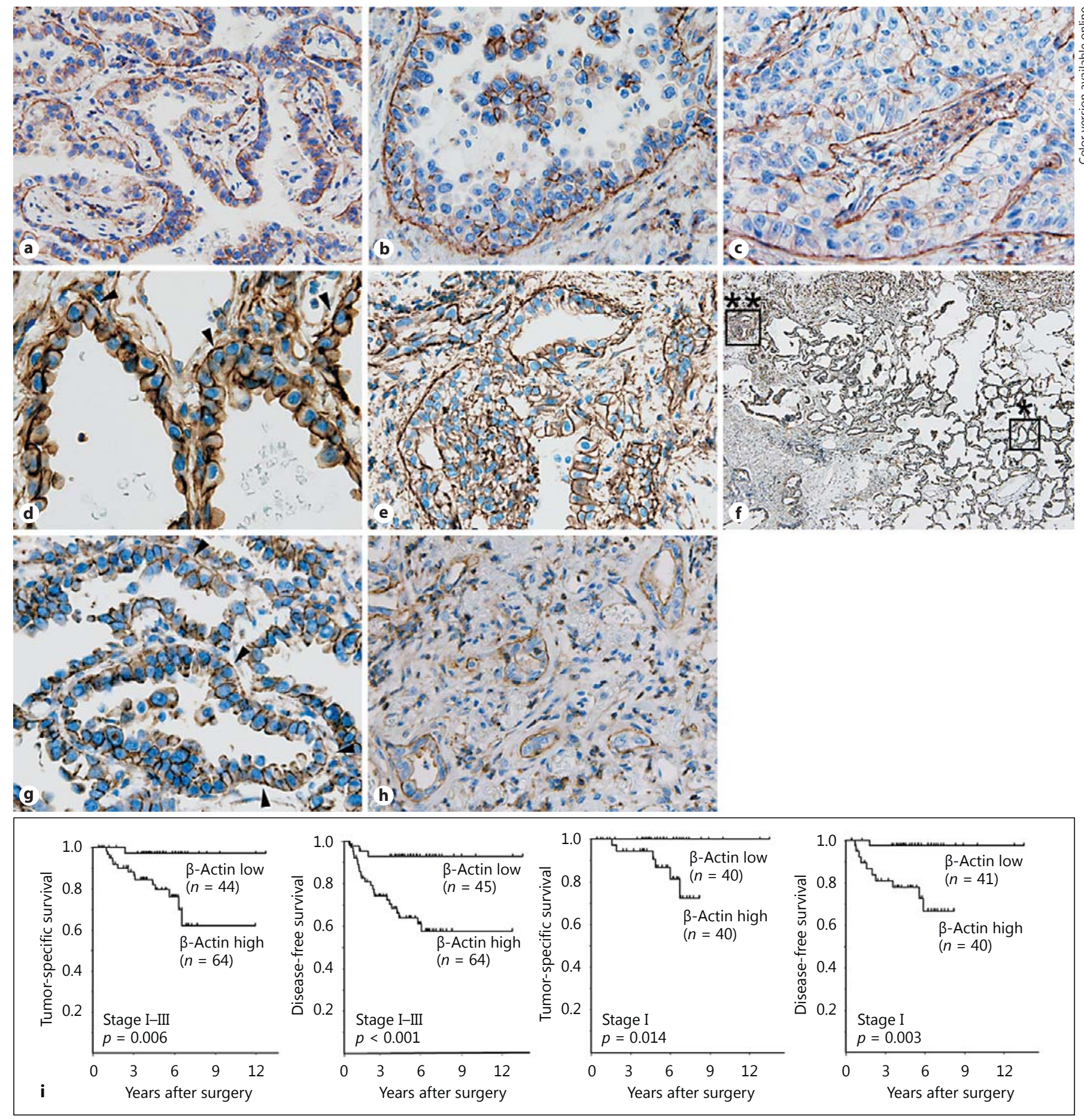

Fig. 2. Subcellular localization of $\beta$-actin in invasive adenocarcinomas and its significance in prognosis. Invasive papillary (a), micropapillary (b), and solid (c) adenocarcinomas. Lepidic/noninvasive area (d) and invasive area (e) of microinvasive adenocarcinoma (f). $\mathbf{d}$ and $\mathbf{e}$ show higher magnification of the boxed areas $*$ and ${ }^{* *}$ in $\mathbf{f}$, respectively. Invasive lepidic adenocarcinoma lep$\mathrm{idic} /$ noninvasive area $(\mathbf{g})$ and invasive area (h). Arrowheads in $\mathbf{d}$ and $\mathbf{g}$ show occasional localization of $\beta$-actin to the matrix-side cytoplasm of lepidic cancer cells. Original magnification, $\times 160$ $(\mathbf{a}-\mathbf{c}, \mathbf{e}, \mathbf{h}), \times 240(\mathbf{d}, \mathbf{g}), \times 14$ (f). i Tumor-specific and disease-free Kaplan-Meier survival curves for the matrix-side localization of $\beta$-actin "high" and "low" groups. $p$ values were calculated by logrank test. 
Table 1. Correlation between degree of matrix-side localization of $\beta$-actin and clinicopathological parameters

\begin{tabular}{|c|c|c|c|}
\hline & \multicolumn{3}{|l|}{$\beta$-Actin ${ }^{\mathrm{a}}$} \\
\hline & $\begin{array}{l}\text { Low } \\
(n=44)\end{array}$ & $\begin{array}{l}\text { High } \\
(n=65)\end{array}$ & $p$ \\
\hline Tumor size, $\mathrm{cm}$ & $2.4 \pm 1.5$ & $2.7 \pm 1.2$ & $0.26^{\mathrm{b}}$ \\
\hline p stage, $n$ & & & $<0.001^{\mathrm{c}}$ \\
\hline I & 41 & 40 & \\
\hline II & 2 & 10 & \\
\hline III & 1 & 15 & \\
\hline $\mathrm{p} \mathrm{N}, n$ & & & $<0.001^{\mathrm{c}}$ \\
\hline No & 42 & 42 & \\
\hline N1 & 2 & 8 & \\
\hline $\mathrm{N} 2, \mathrm{~N} 3$ & 0 & 15 & \\
\hline Lymphatic permeation, $n$ & & & $<0.001^{\mathrm{c}}$ \\
\hline- & 41 & 34 & \\
\hline+ & 3 & 31 & \\
\hline Vascular permeation, $n$ & & & $<0.001^{\mathrm{c}}$ \\
\hline- & 37 & 33 & \\
\hline+ & 7 & 32 & \\
\hline Laminin, $n$ & & & $<0.001^{\mathrm{c}}$ \\
\hline Low & 31 & 5 & \\
\hline High & 13 & 60 & \\
\hline Histological subtype, $n$ & & & $<0.001^{\mathrm{C}}$ \\
\hline AIS/MIA & 32 & 7 & \\
\hline Invasive & 13 & 57 & \\
\hline Lepidic adenocarcinoma & 6 & 13 & \\
\hline Acinar adenocarcinoma & 2 & 8 & \\
\hline Papillary adenocarcinoma & 2 & 14 & \\
\hline Micropapillary adenocarcinoma & 0 & 3 & \\
\hline Solid adenocarcinoma & 1 & 16 & \\
\hline Invasive mucinous & & & \\
\hline adenocarcinoma & 2 & 3 & \\
\hline
\end{tabular}

${ }^{\text {a }}$ Matrix-side localization. ${ }^{\mathrm{b}}$ Welch $t$ test. ${ }^{\mathrm{c}}$ Mann-Whitney U test.

staining on a paraffin section of Lifeact-expressing A549 cells were similar (online suppl. Fig. 2E, F). Accordingly, we concluded that the $\beta$-actin immunostaining in this study represented F-actin.

\section{Clinical Significance of the Matrix-Side Cortical Actin} of Lung Adenocarcinoma Cells

We identified 65 out of 109 adenocarcinoma cases that were estimated to have a high degree of matrix-side localization of $\beta$-actin. The degree of matrix-side localization of $\beta$-actin was correlated with tumor extension, lymph node metastasis, vascular permeation, and lymphatic permeation (Table 1).

Tumor-specific survival and disease-free survival of both the stage I and stage I-III patients were significantly worse for the group in which the matrix-side localization of $\beta$-actin was high compared to the low group (Fig. 2i). Multivariate analysis showed a trend toward worse prognosis of the $\beta$-actin high group (online suppl. Table 2). These results suggest a close relationship between the matrix-side localization of $\beta$-actin with invasion and metastasis.

\section{Cortical F-Actin Distribution in 3D-Cultured A549 Cells}

In order to clarify the significance of cortical F-actin in cancer cell invasion, we first studied the distribution of F-actin in lung adenocarcinoma A549 cells in a 3D environment. When lung adenocarcinoma A549 cells were grown in type I collagen and basement membrane extract Matrigel mixed gel [5] for 8 days, A549 cells formed round spheroids without a luminal structure. By phalloidin staining, cortical F-actin was mainly distributed beneath the matrix-side plasma membrane and faintly beneath the intercellular plasma membrane in almost all of the A549 spheroids (Fig. 3a).

Our study of clinical materials revealed a close correlation between the matrix-side cortical actin and the loss of basement membrane laminin- $\mathrm{C} 1$, and this suggested a causal relationship between them. Accordingly, we studied the effect of basement membrane extract Matrigel on matrix-side F-actin. In pure Matrigel, the matrix-side cortical F-actin was less intense than those in a type I collagen and Matrigel mixed gel (Fig. 3a). Phosphorylated MLC, which regulates F-actin contraction, was also observed at the matrix-side in a type I collagen and Matrigel mixed gel, and, similarly to F-actin, it was less intense in pure Matrigel (Fig. 3b; online suppl. Fig. 3). Using the myosin inhibitor blebbistatin, which suppresses actomyosin contraction through the inhibition of myosin ATPase activity [15], matrix-side cortical F-actin became sparse and unclear (Fig. 3c).

By super-resolution and electron microscopy, the matrix-side surface of A549 cells in type I collagen and Matrigel mixed gels showed the wrinkling of plasma membrane underlaid by F-actin (Fig. 4a). With the addition of blebbistatin, the surface wrinkling was stretched and expanded. The matrix-side surface of A549 cells in pure Matrigel was nearly smooth and F-actin was partly discontinuous. In the surgically resected lung adenocarcinoma cells, the matrix-side surface was mostly smooth but wrinkles were observed occasionally (Fig. 4b).

These results suggest that compact wrinkling of the plasma membrane by myosin-mediated F-actin contraction is one explanation for cortical actin accumulation at
176

Pathobiology 2017;84:171-183

DOI: $10.1159 / 000452838$
Tomii et al. 


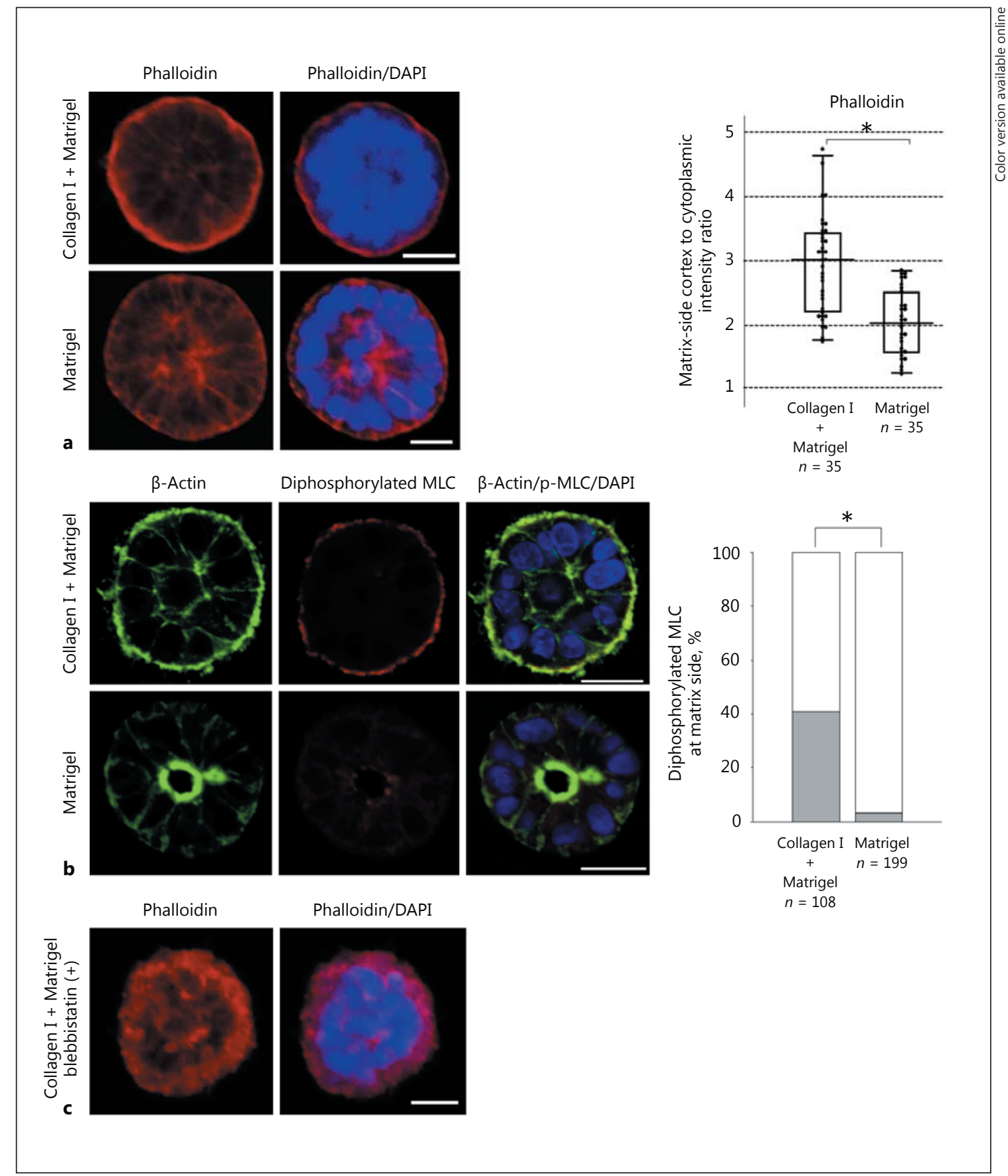

Fig. 3. Cortical F-actin distribution in 3D-cultured A549 cells and effects of basement membrane extract Matrigel and blebbistatin. a F-actin localization. A549 cells were cultured in a type I collagen and Matrigel mixed gel or in pure Matrigel for 8 days, fixed with paraformaldehyde, and stained with phalloidin-Alexa 555 and DAPI. The values shown for phalloidin are intensity cortex $_{\text {intensi- }}$ ty cytoplasm. $^{*} p<0.001$, Mann-Whitney test. b $\beta$-Actin and phos- phorylated MLC localization of A549 cells cultured in a type I collagen and Matrigel mixed gel or in pure Matrigel. Both were immunostained for paraffin-embedded A549 cells. ${ }^{*} p<0.001$, Mann-Whitney test. c Effect of blebbistatin on F-actin. A549 cells cultured in a type I collagen and Matrigel mixed gel were incubated with blebbistatin $(50 \mu \mathrm{M})$ for last $45 \mathrm{~h}$. Scale bar, $10 \mu \mathrm{m}$. 


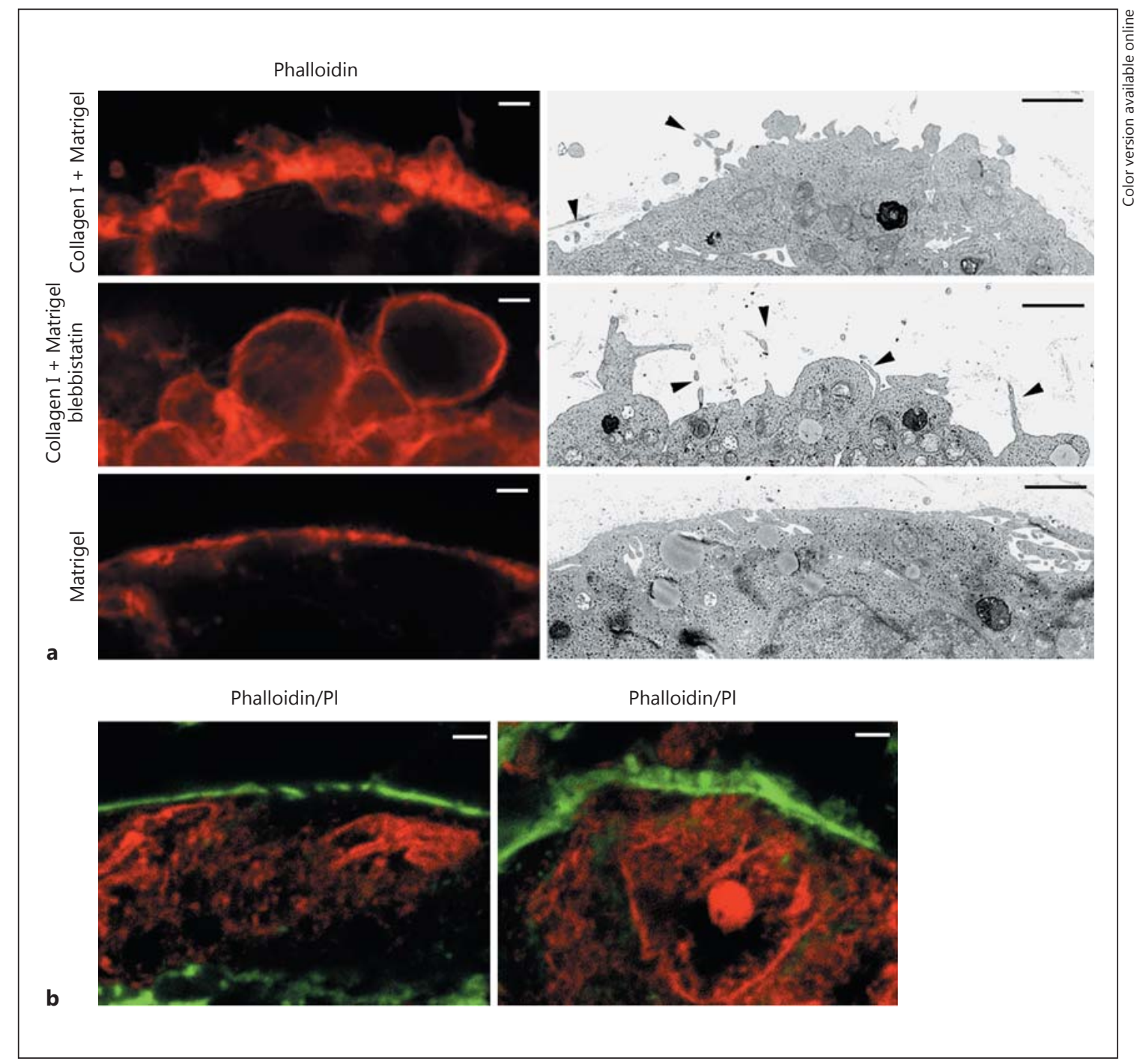

Fig. 4. Super-resolution microscopy and electron microscopy findings of matrix-side surface of lung adenocarcinoma cells. a Super-resolution microscopy findings (left) and electron microscopy findings (right) of A549 cells in a type I collagen and Matrigel mixed gel without or with blebbistatin, and in pure Matrigel. Factin was stained with phalloidin-Alexa 555. Arrowheads show cy-

the matrix-side cytoplasm. The basement membrane was considered to be a regulator of matrix-side cortical actin accumulation through suppression of MLC phosphorylation.

\section{Participation of Cortical F-Actin in 3D Migration of} A549 Cells

Next, we examined whether cortical F-actin participates in the 3D migration of A549 cells. Cdc42 possibly participates in 2D [16], amoeboid [17], and collective mi- toplasmic spikes. b Super-resolution microscopy findings of smooth surface (left) and wrinkling surface (right) of adenocarcinoma cells in surgically resected lung tissue. F-actin was stained with phalloidin-Alexa 488 . The matrix side is to the top. Scale bar, $2 \mu \mathrm{m}$. PI, propidium iodide.

gration [5], as well as matrix proteolysis through invadopodia induction [18]. In addition, vav1 [18], a possible Cdc42 activator [18], is frequently overexpressed in lung cancers [19], which suggests a possible Cdc42 participation in these cancers. Accordingly, we transduced constitutively active Cdc42 to A549 cell spheroids by lentiviral vectors in order to migrate them. In addition, MT1-MMP was simultaneously expressed because endogenous expression of MT1-MMP in A549 cells was about one 200th of that in normal small airway epithelial cells by RT-PCR 
Fig. 5. Cortical F-actin distribution in a $3 \mathrm{D}$ migration model of A549 cells. A 3D-reconstructed images of A549 cell colonies after cotransduction with constitutively active Cdc42 and MT1-MMP. A549 cells were cultured in a type I collagen and Matrigel mixed gel for 5 days, constitutively active Cdc42 and MT1-MMP were lentivirally transduced for $15 \mathrm{~h}$, and the cells were incubated for an additional 25-45 h (40-60 h after transduction). B Comparison of cortical F-actin intensity of protruding parts and spheroid parts of A549 cell colonies $40 \mathrm{~h}$ after transduction. The value shown is (intensity cortex $_{\text {intensity }}$ cytoplasm $_{\text {) }}$ of the protruding part/(intensity cortex $_{\text {in }}$ tensity cytoplasm $_{\text {) of the spheroid part. }{ }^{*} p<}$ 0.001 , paired $t$ test, $n=35$. Cortical F-actin of the protruding part $(\mathrm{a}, \mathrm{b})$ and spheroid part (c) by super-resolution microscopy. Scale bar, $2 \mu \mathrm{m}$. Arrowheads show a few plasma membrane wrinkles existing in the protruding cells.

Cortical Actin in Lung Adenocarcinoma
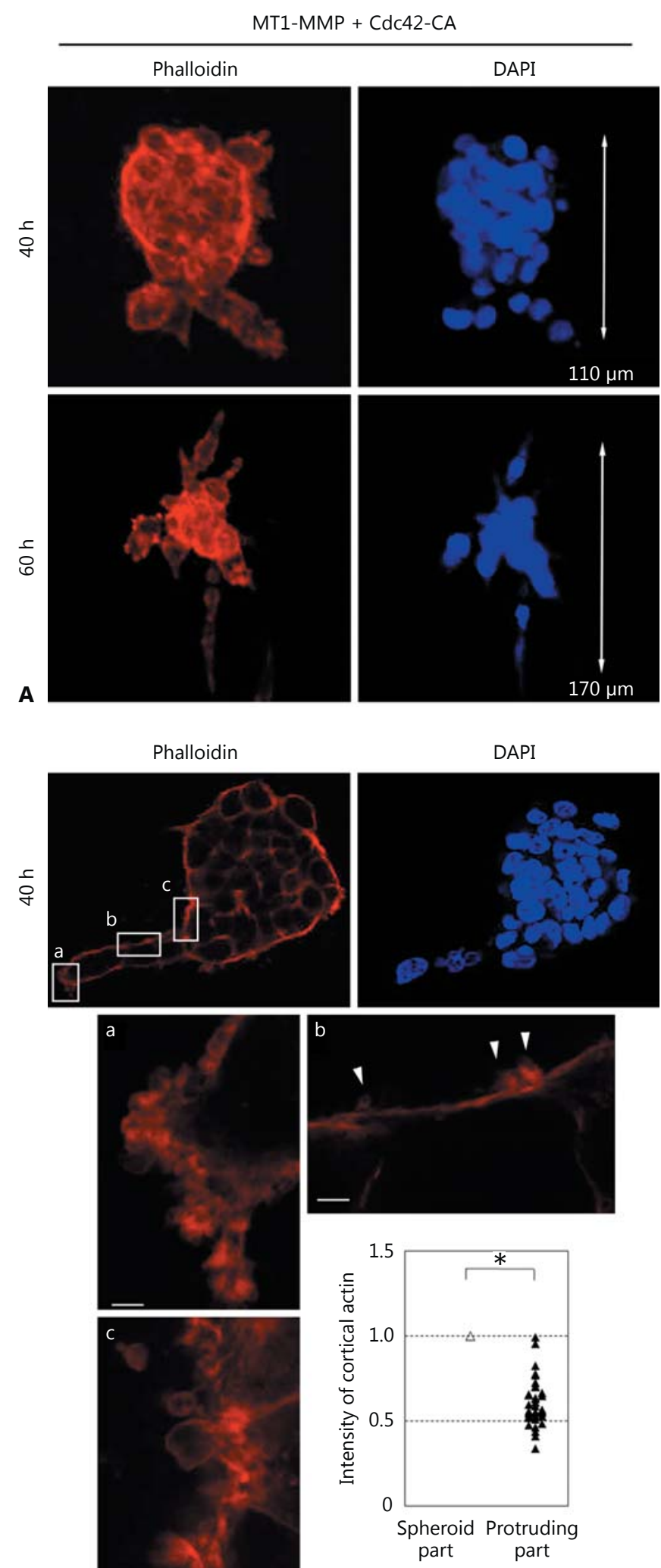

B

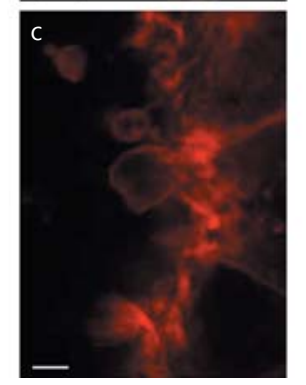




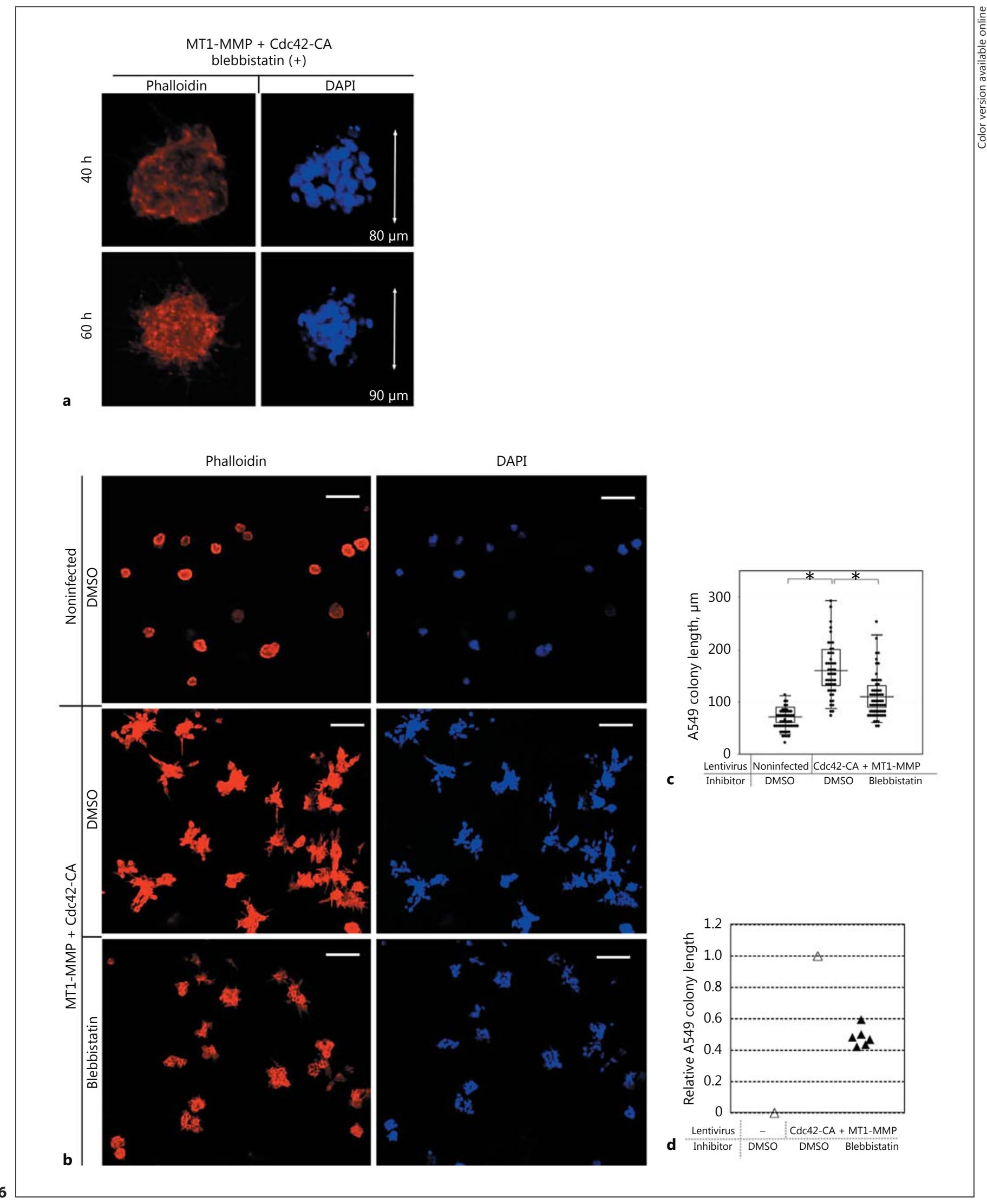

(For legend see next page.) 
quantification (data not shown). As expected, about 40 $60 \mathrm{~h}$ after the lentiviral transduction of both the genes, chains of A549 cells were protruding from the spheroids into the matrix (Fig. 5A). The transduction of constitutively active $\mathrm{Cdc} 42$ alone was partially effective on migration, but dominant negative $\mathrm{Cdc} 42$ was not effective at all (online suppl. Fig. 4).

The matrix-side cortical F-actin of the protruding cells was less intense than that of the spheroid parts $(0.56: 1$, $n=35$, paired $t$ test, $p<0.001)$ and the cells with attenuated cortical F-actin protruded into the matrix. By superresolution microscopy, the matrix-side surface of the protruding cells was mostly smooth with a few wrinkles reflecting less intense cortical F-actin. In contrast, prominent plasma membrane wrinkles at the spheroid parts and cytoplasmic protrusions at the leading edge were observed (Fig. 5B).

We examined whether inhibition of cortical F-actin contraction by myosin inhibitor blebbistatin affected the $3 \mathrm{D}$ migration of A549 cells. Under the existence of blebbistatin, the A549 cells transduced with active Cdc42 and MT1-MMP showed fine spikes, about 10-30 $\mu \mathrm{m}$ in length, on the surface of the spheroids, as were previously described in colon cancer Caco-2 cells [20]. However, their nuclei remained behind (Fig. 6a, b). When the lengths of colonies were measured at the nuclear position, they were elongated 2.1 -fold by the simultaneous transduction of constitutively active Cdc42 and MT1MMP. This elongation was significantly suppressed to $48 \%$ by blebbistatin (Fig. 6c, d). This suggests that cortical F-actin and phosphorylated myosin, both of which

Fig. 6. Effect of blebbistatin on 3D migration of A549 cells. a, b High and low magnification of 3D migrating A549 cells with or without blebbistatin. A549 cells were cultured in a type I collagen and Matrigel mixed gel for 5 days, constitutively active Cdc42 and MT1-MMP were lentivirally transduced for $15 \mathrm{~h}$, and the cells were incubated for an additional 25-45 h with vehicle/DMSO or with blebbistatin $(50 \mu \mathrm{M})$. Scale bar, $200 \mu \mathrm{m}$. c Effect of blebbistatin on the 3D migration of A549 cells represented by the maximum axial length of 3D-cultured A549 colonies. The result of 1 representative experiment is shown. More than 50 colonies per each condition were measured. ${ }^{*} p<0.001$, Mann-Whitney test. d Reproducibility of 6 independent experiments. Average axial length of 3D-cultured A549 cell colonies with cotransduction of active Cdc42 and MT1-MMP and blebbistatin was normalized to that of a negative control without transduction, and to a positive control with cotransduction of active Cdc42 and MT1-MMP and vehicle/ DMSO. The value shown is (length blebbistatin $_{\text {- length }}$ negative control $) /$ (length positive control $_{-}$length negative control $_{\text {). }}$.

Cortical Actin in Lung Adenocarcinoma are mainly located in the matrix side of A549 colonies, promotes $3 \mathrm{D}$ migration through myosin-mediated cortical F-actin contraction.

In order to exclude the effect of proliferating activity on the axial length of colonies, we quantified the growth of A549 cells with lentiviral gene transduction (online suppl. Fig. 5). Constitutively active Cdc42 suppressed the growth of A549 cells to one-third and blebbistatin restored the cell growth suppressed by active Cdc42. These results suggest that the axial length does not reflect alteration of the proliferating activity by the transduction of constitutively active Cdc42 or blebbistatin.

\section{Discussion}

We showed that cortical actin accumulated at the matrix-side cytoplasm in invasive lung adenocarcinoma cells. The source of cortical actin accumulation at the matrix-side cytoplasm of invasive adenocarcinoma cells can be explained by 2 possibilities: compact wrinkling of the plasma membrane by myosin-mediated contraction as well as an increased amount of cortical F-actin due to either promoted assembly or suppressed disassembly.

The super-resolution and electron microscopy studies of A549 cells suggest that accumulation at the matrix-side cortical actin reflects plasma membrane wrinkling. Plasma membrane wrinkles are unfolded by increased mechanical tension of the plasma membrane [21], which is negatively regulated by actomyosin contraction [22] Conversely, it can be said that plasma membrane wrinkling is promoted by contraction of the underlying cortical actin [23]. The existence of phosphorylated MLC with F-actin and expanded wrinkles by the addition of blebbistatin, the inhibitor of actomyosin contraction, support myosin-mediated plasma membrane wrinkling.

The polarized distribution of F-actin in the epithelial cells is dependent on Racl activation and RhoA inactivation [24, 25]. Conversely, polarity inversion occurs through the signal from activated RhoA that leads to MLC phosphorylation $[25,26]$. Laminin, the major component of basement membrane, preferentially activates Rac1 over RhoA in A549 cells [27]. Additionally, MLC phosphorylation at the matrix side of A549 cells was diminished in pure Matrigel. These findings suggest that the suppression of RhoA to myosin signaling by the basement membrane can explain the relationship between basement membrane composition and the matrix-side cortical actin accumulation in both clinical samples and 3D cultured A549 cells.

Pathobiology 2017;84:171-183 DOI: $10.1159 / 000452838$ 
An increased amount of cortical F-actin due to either promoted assembly or suppressed disassembly is another possible explanation for accumulation of the matrix-side cortical actin. One of the regulators of cortical F-actin assembly and disassembly is $\operatorname{PtdIns}(4,5) \mathrm{P}_{2}$ [28] by its activation of actin nucleators, such as mDia1 [29] and inhibition of actin depolymerizing proteins, such as cofilin [30]. PtdIns $(4,5) \mathrm{P}_{2}$ and F-actin are mainly located in the apical portion of polarized epithelium and the putative determinant of this orientation is also the signaling that originated from the binding of basement membrane laminin with its integrin receptor and dystroglycan $[24,26]$. Accordingly, PtdIns-mediated F-actin regulation is another possible explanation for the accumulation of the matrixside cortical actin and its relationship with loss of the basement membrane.

In this study, the migration of A549 cells was significantly suppressed under the presence of myosin inhibitor blebbistatin. This suggests that cortical F-actin and phosphorylated myosin, both of which are mainly located in the matrix side of A549 colonies, promotes 3D migration through myosin-mediated cortical F-actin contraction. Previous studies also showed that the collective migration of squamous cell carcinoma A431 cells is suppressed by the reduction of MLC phosphorylation, which was localized to the cortex in contact with matrix [5], and this also suggests participation of the matrix-side cortical actin contraction in cancer cell migration. Nuclear translocation in the 3D migration of leukocytes is dependent on cortical actin contraction [31] and it might be hypothesized that cortical actin contraction is necessary for the translocation of intracytoplasmic components, also including the nucleus in the collective migration of cancer cells. However, it remains to be clarified whether subcellular localization of cortical actin to the matrix side is crucial for $3 \mathrm{D}$ collective migration. The reproducibility of the experiment using other cell lines is another subject that remains to be clarified.

In spite of the existence of matrix-side cortical F-actin and phosphorylated myosin, A549 cells in 3D gels formed expansive spheroids without protruding cells to the surrounding matrix. Thus, the existence of matrix-side cortical F-actin alone is presumably not sufficient for collective cancer cell migration. Leader cells in collective migration show prominent actin polymerization, which forms filopodia or pseudopodia [32], and suppressed actomyosin contraction [33], which negatively regulates the initiation of leader cells [34]. Attenuated cortical F-actin and stretched plasma membrane of protruding A549 cells induced by active Cdc42 and MT1-MMP transduction sug- gests actin polymerization beyond the cortical actin contraction, and a similar characteristic to leader cells in collective migration. Accordingly, it can be assumed that collective migration requires coordination of cortical Factin contraction and actin polymerization toward the direction of migration. Cdc42 possibly participates in actin polymerization beyond the cortical actin contraction in the protruding cells through N-WASP/ARP2 activation [18].

This investigation exclusively focused on lung adenocarcinomas; however, our preliminary study revealed that matrix-side localization of cortical actin was also found in squamous cell carcinomas and small cell carcinomas (online suppl. Fig. 6). Accordingly, it is possible to say that cortical actin participates in the invasion of lung cancers other than adenocarcinomas.

Alteration of cortical actin localization can be regarded as axial inversion in invasive cancer cells. Axial inversion was previously reported in cancers of other organs, such as breast [35], gastric [36], salivary [37], and urothelial cancers [38], as micropapillary subtypes with their aggressive characteristics. It is possible that translocation of apical surface characteristics to the matrix side occurs even in the ordinary subtypes of invasive cancers, and some of the translocated molecules possibly participate in cancer cell invasion.

In conclusion, our studies revealed actin accumulation at the matrix-side cortex in invasive lung adenocarcinoma cells, as well as its correlation with tumor stages and poor prognosis. Contraction of cortical F-actin in the cellular collectives possibly participates in the migration of cancer cells.

\section{Acknowledgement}

The authors thank Masaki Sekine, Takahiro Sekiya, Shinya Ayabe, Yuriko Wada, and Shizuko Ichinose for their technical assistance, Naoyuki Fujiwara and Kenichi Ohkubo for providing clinical information and requisition of informed consent. This research was supported by a grant-in-aid from the Ministry of Education, Science, Sports and Culture of Japan (22590309, 25460433).

\section{Disclosure Statement}

The authors have no conflict of interest to declare.
182

Pathobiology 2017;84:171-183

DOI: $10.1159 / 000452838$
Tomii et al. 


\section{References}

1 Pollard TD, Borisy GG: Cellular motility driven by assembly and disassembly of actin filaments. Cell 2003;112:453-465.

2 Friedl P, Wolf K: Tumour-cell invasion and migration: diversity and escape mechanisms. Nat Rev Cancer 2003;3:362-374.

3 Paluch EK, Raz E: The role and regulation of blebs in cell migration. Curr Opin Cell Biol 2013;25:582-590.

4 Poincloux R, Collin O, Lizarraga F, Romao M, Debray M, Piel M, Chavrier P: Contractility of the cell rear drives invasion of breast tumor cells in 3D matrigel. Proc Natl Acad Sci USA 2011;108:1943-1948.

5 Gaggioli C, Hooper S, Hidalgo-Carcedo C, Grosse R, Marshall JF, Harrington K, Sahai E: Fibroblast-led collective invasion of carcinoma cells with differing roles for RhoGTPases in leading and following cells. Nat Cell Biol 2007;9:1392-1400.

6 Bovellan M, Romeo Y, Biro M, Boden A, Chugh P, Yonis A, Vaghela M, Fritzsche M, Moulding D, Thorogate R, Jegou A, Thrasher AJ, Romet-Lemonne G, Roux PP, Paluch EK, Charras G: Cellular control of cortical actin nucleation. Curr Biol 2014;24:1628-1635.

7 Salbreux G, Charras G, Paluch E: Actin cortex mechanics and cellular morphogenesis. Trends Cell Biol 2012;22:536-545.

8 Grego-Bessa J, Hildebrand J, Anderson KV: Morphogenesis of the mouse neural plate depends on distinct roles of cofilin 1 in apical and basal epithelial domains. Development 2015;142:1305-1314

9 Baum B, Perrimon N: Spatial control of the actin cytoskeleton in Drosophila epithelial cells. Nat Cell Biol 2001;3:883-890.

10 Blanchard GB, Murugesu S, Adams RJ, Martinez-Arias A, Gorfinkiel N: Cytoskeletal dynamics and supracellular organisation of cell shape fluctuations during dorsal closure. Development 2010;137:2743-2752.

11 Hirooka S, Akashi T, Ando N, Suzuki Y, Ishida N, Kurata M, Takizawa T, Kayamori K, Sakamoto K, Fujiwara N, Kojima M, Eishi Y: Localization of the invadopodia-related proteins actinin- 1 and cortactin to matrix-contact-side cytoplasm of cancer cells in surgically resected lung adenocarcinomas. Pathobiology 2011;78:10-23.

12 Travis WD, Brambilla E, Burke AP, Marx A, Nicholson AG (eds): WHO Classification of Tumours of the Lung, Pleura, Thymus and Heart, ed 4. Geneva, WHO Press, 2015.

13 Goldstraw P: Staging Manual in Thoracic Oncology. Orange Park, Editorial Rx Press, 2009.

14 Riedl J, Crevenna AH, Kessenbrock K, Yu JH, Neukirchen D, Bista M, Bradke F, Jenne D, Holak TA, Werb Z, Sixt M, Wedlich-Soldner R: Lifeact: a versatile marker to visualize Factin. Nat Methods 2008;5:605-607.
15 Straight AF, Cheung A, Limouze J, Chen I, Westwood NJ, Sellers JR, Mitchison TJ: Dissecting temporal and spatial control of cytokinesis with a myosin II inhibitor. Science 2003;299:1743-1747.

16 Etienne-Manneville S, Hall A: Integrin-mediated activation of $\mathrm{Cdc} 42$ controls cell polarity in migrating astrocytes through PKC. Cell 2001;106:489-498.

17 Gadea G, Sanz-Moreno V, Self A, Godi A, Marshall CJ: DOCK10-mediated Cdc42 activation is necessary for amoeboid invasion of melanoma cells. Curr Biol 2008; 18:14561465.

18 Razidlo GL, Schroeder B, Chen J, Billadeau DD, McNiven MA: Vav1 as a central regulator of invadopodia assembly. Curr Biol 2014; 24:86-93.

19 Lazer G, Idelchuk Y, Schapira V, Pikarsky E, Katzav S: The haematopoietic specific signal transducer Vavl is aberrantly expressed in lung cancer and plays a role in tumourigenesis. J Pathol 2009;219:25-34.

20 Ivanov AI, Hopkins AM, Brown GT, GernerSmidt K, Babbin BA, Parkos CA, Nusrat A: Myosin II regulates the shape of three-dimensional intestinal epithelial cysts. J Cell Sci 2008;121:1803-1814.

21 Figard L, Xu H, Garcia HG, Golding I, Sokac AM: The plasma membrane flattens out to fuel cell-surface growth during Drosophila cellularization. Dev Cell 2013;27:648-655.

22 Gauthier NC, Fardin MA, Roca-Cusachs P, Sheetz MP: Temporary increase in plasma membrane tension coordinates the activation of exocytosis and contraction during cell spreading. Proc Natl Acad Sci USA 2011;108: 14467-14472.

23 Gabriele S, Benoliel AM, Bongrand P, Theodoly O: Microfluidic investigation reveals distinct roles for actin cytoskeleton and myosin II activity in capillary leukocyte trafficking. Biophys J 2009;96:4308-4318.

24 Yu W, Datta A, Leroy P, O’Brien LE, Mak G, Jou TS, Matlin KS, Mostov KE, Zegers MM: $\beta 1$-integrin orients epithelial polarity via Rac1 and laminin. Mol Biol Cell 2005;16:433-445.

$25 \mathrm{Yu} \mathrm{W}$, Shewan AM, Brakeman P, Eastburn DJ, Datta A, Bryant DM, Fan QW, Weiss WA, Zegers MM, Mostov KE: Involvement of RhoA, ROCK I and myosin II in inverted orientation of epithelial polarity. EMBO Rep 2008;9:923-929.

26 O'Brien LE, Jou TS, Pollack AL, Zhang Q, Hansen SH, Yurchenco P, Mostov KE: Rac1 orientates epithelial apical polarity through effects on basolateral laminin assembly. Nat Cell Biol 2001;3:831-838.
27 Gu J, Sumida Y, Sanzen N, Sekiguchi K: Laminin-10/11 and fibronectin differentially regulate integrin-dependent Rho and Rac activation via p130 Cas_-CrkII-DOCK180 pathway. J Biol Chem 2001;276:27090-27097.

28 Saarikangas J, Zhao H, Lappalainen P: Regulation of the actin cytoskeleton-plasma membrane interplay by phosphoinositides. Physiol Rev 2010;90:259-289.

29 Rousso T, Shewan AM, Mostov KE, Schejter ED, Shilo BZ: Apical targeting of the formin diaphanous in drosophila tubular epithelia. eLife 2013;2:e00666.

30 van Rheenen J, Song X, van Roosmalen W Cammer M, Chen X, Desmarais V, Yip SC, Backer JM, Eddy RJ, Condeelis JS: EGF-induced $\mathrm{PIP}_{2}$ hydrolysis releases and activates cofilin locally in carcinoma cells. J Cell Biol 2007;179:1247-1259.

31 Lammermann T, Bader BL, Monkley SJ, Worbs T, Wedlich-Soldner R, Hirsch K, Keller M, Forster R, Critchley DR, Fassler R, Sixt M: Rapid leukocyte migration by integrin-independent flowing and squeezing. $\mathrm{Na}-$ ture 2008;453:51-55.

32 Friedl P, Gilmour D: Collective cell migration in morphogenesis, regeneration and cancer. Nat Rev Mol Cell Biol 2009;10:445-457.

33 Omelchenko T, Vasiliev JM, Gelfand IM, Feder HH, Bonder EM: Rho-dependent formation of epithelial "leader" cells during wound healing. Proc Natl Acad Sci USA 2003; 100:10788-10793.

34 Reffay M, Parrini MC, Cochet-Escartin O, Ladoux B, Buguin A, Coscoy S, Amblard F, Camonis J, Silberzan P: Interplay of Rhoa and mechanical forces in collective cell migration driven by leader cells. Nat Cell Biol 2014;16: 217-223.

35 Siriaunkgul S, Tavassoli FA: Invasive micropapillary carcinoma of the breast. Modern Pathol 1993;6:660-662.

36 Roh JH, Srivastava A, Lauwers GY, An J, Jang KT, Park CK, Sohn TS, Kim S, Kim KM: Micropapillary carcinoma of stomach: a clinicopathologic and immunohistochemical study of 11 cases. Am J Surg Pathol 2010;34:11391146.

37 Nagao T, Gaffey TA, Visscher DW, Kay PA, Minato H, Serizawa H, Lewis JE: Invasive micropapillary salivary duct carcinoma: a distinct histologic variant with biologic significance. Am J Surg Pathol 2004;28:319-326.

38 Samaratunga $\mathrm{H}$, Khoo K: Micropapillary variant of urothelial carcinoma of the urinary bladder: a clinicopathological and immunohistochemical study. Histopathology 2004;45: 55-64. 